\title{
Maintaining Traction Factor Value Of Belt Conveyor With Two-Engine Drive
}

\author{
[Kaung Pyae Aung]
}

\begin{abstract}
The paper deals with maintaining traction factor value of belt conveyor with two-engine drive. The system which maintains traction factor value by changing the weight of tensioning device of belt conveyor has been developed for this task. The results of computer modeling in SIMULINK were transient in velocity of concentrated masses of belt, moving the tensioning device and dynamics of traction factor in the implementation of the control of carriage provisions of tensioning device.
\end{abstract}

Keywords: belt conveyor, simulation, automatic control system of the speed of conveyor belt, traction factor and transients.

\section{Introduction}

The increase of factory automation has been an important factor in worldwide industrial progress during the past several decades. Manufacturing lines in industrial plants typically involve one or more variable speed motor drives that serve to power conveyor belts, robot arms, paper mills, plastic and fiber processing lines.

A typical conveyor system has diverse applications largely in industries, and the range of diversity depends on function, for example transportation of materials, workmanship, loading goods, passenger transportation i.e. at airport, luggage conveying and so on. Conveyor designs comes in variety of forms long or short horizontal conveying, inclined or declined conveying and vertical conveying, dependent much on the location or area of work. Belt conveyors are economical, efficient and are a more and more often used means of transportation. Advancements in belt conveyor technology have provided essentially unlimited adaptability, allowing conveyors to provide solutions for every geographic region and all belts of applications worldwide.

\section{System modelling}

- Design scheme of the conveyor system with two-engine drive and the tensioning device in the tail section is shown in Figure 1. A system with distributed parameters is approximated by six concentrated masses, three of them $m_{1}, m_{2}, m_{3}$ are located on the loading branch, two $m_{4}, m_{5}$ on the empty branch and $m_{6}$ is represented as a mass of tensioning device. Coordinates of the five masses $m_{1}, m_{2}, m_{3}, m_{4}, m_{5}$, their velocities $\dot{s}_{1}, \dot{s}_{2}, \dot{s}_{3}, \dot{s}_{4}, \dot{s}_{5}, \dot{s}_{6}, \quad$ their movements $s_{1}, s_{2}, s_{3}, s_{4}, s_{5}, s_{6}$, point and movement velocity of tension device. Finite-dimensional mathematical model of the conveyor with a load described fourteen coordinates state $S=\left(s_{1}, s_{2}, s_{3}, s_{4}, s_{5}, s_{6}, s_{7}, \dot{s}_{1}, \dot{s}_{2}, \dot{s}_{3}, \dot{s}_{4}, \dot{s}_{5}, \dot{s}_{6}, \dot{s}_{7}\right)^{T}$.

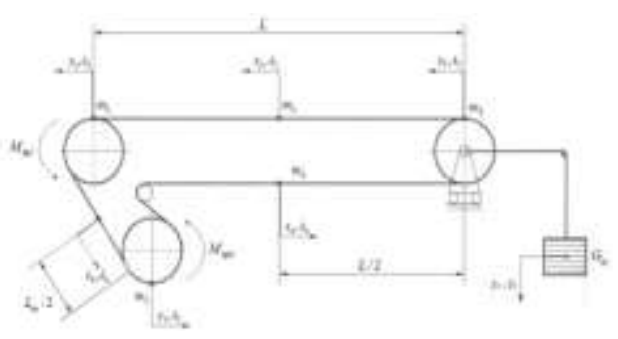

Fig.1. Design scheme conveyor with two-engine drive

We have the case of the software SIMULINK, MATLAB which has a power system toolbox for the study of electrical machines. The problem is related to the stabilization of the value of the traction factor. Improving the efficiency of operation of the conveyor belt is more concerned with the reduction of wear of the moving web. Slippage may occur by increasing the speed of the drive drum. Slippage effect occurs when the value of the traction factor exceeds the value that can be 
called critical. The value of traction factor is calculated as follows:

$$
E^{\mu \alpha}(t)=\frac{S_{4}(t)}{S_{1}(t)}
$$

where $S_{4}$ - the tension on the incoming branch, $S_{1}$ - the tension on the trailing branch.

If single-engine belt conveyor with an angle of coverage of the tape of the roller $\alpha=\pi$, there will be no slippage under the condition $E^{\mu \alpha} \leq 2,5$. In the case of two-engine drive the common traction factor is the product of tractive factors on each of the drive drums. Therefore, it should be $E^{\mu \alpha} \leq 6,25$. If we achieved maintaining common traction factor is not above this level, we can reduce the risk of slippage on each individual drive drum.

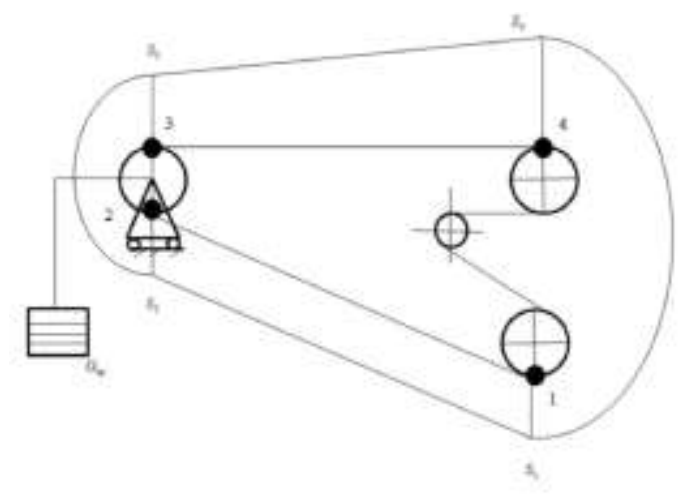

Fig.2. Diagram of tension for conveyor with tensioning device

We use the relationship between its size and the tensioning device weight of the conveyor for the development of the stabilization system of the traction factor. For traction factor, we must know the tension on the trailing branch $S_{l}$ and the tension on the incoming branch $S_{4}$. The stretching portions of the tape is also changing during the change of the speed of the conveyor. Let $\Delta_{1}=s_{4}-s_{3}$ is the stretch of empty branch and $\Delta_{4}=s_{1}-s_{2}$ is the stretch of tensions on the loading branch. Deformations $\Delta_{1}$ and $\Delta_{4}$ can change during the change of tensioning device weight. And the tensions values $S_{1}$ and $S_{4}$ depend on this deformations. Tensions can be calculated according to the formulas:

$$
\begin{aligned}
& S_{1}=0,5 G_{\mu y}-W_{2,1}, \\
& S_{4}=0,5 G_{\mu y}+W_{4,3},
\end{aligned}
$$

Where $W_{2,1}$ - the resistance movement on the empty branch, $W_{4,3}$ - the resistance movement on the loading branch.

The tensioning device weight changed from 3 tons to 11 tons. These experiments are listed in table 1 .

TABLE 1.

\begin{tabular}{|l|l|l|l|l|}
\hline$G_{H y}$, & $\Delta_{1}, \mathrm{M}$ & $\Delta_{4}, \mathrm{M}$ & $S_{1}, \mathrm{~N}$ & $S_{4}, \mathrm{~N}$ \\
$\mathrm{~N}$ & & & & \\
\hline 30000 & -0.6298 & -0.1337 & 2812,5 & 67500 \\
35000 & -0.5876 & - & 5312.5 & 70000 \\
40000 & -0.5482 & 0.09145 & 7812,5 & 72500 \\
45000 & -0.5071 & -0.0521 & 10312.5 & 75000 \\
52000 & -0.4488 & - & 13812.5 & 78500 \\
60000 & -0.3956 & 0.01092 & 17812.5 & 82500 \\
70000 & -0.3289 & 0.04753 & 22812.5 & 87500 \\
80000 & -0.268 & 0.1006 & 27812.5 & 92500 \\
90000 & -0.2012 & 0.1672 & 32812.5 & 97500 \\
100000 & -0.1377 & 0.2281 & 37812.5 & 102500 \\
110000 & - & 0.2949 & 42812.5 & 107500 \\
& 0.07882 & 0.3585 & & \\
\hline
\end{tabular}

The method of least squares to obtain the dependence of the first order tensions S1 and S4 on the deformation $\Delta_{1}$ and $\Delta_{4}$ :

$$
\begin{aligned}
& S_{1}\left(\Delta_{1}\right)=72990 \Delta_{1}+47525 \\
& S_{4}\left(\Delta_{4}\right)=73138 \Delta_{4}+76004
\end{aligned}
$$

These tensions are not real, because there is an error of approximation. However, with these relationships, it may be possible to obtain a function $E^{\mu \alpha}(t)$ with small error. Model unit will calculate the tension of the formulas (3) and then value of the traction factor of formula (1). Dynamic traction factor is shown in Figure 3. 
Proc. of the Third Intl. Conf. on Advances in Mechanical and Automation Engineering - MAE 2015.

Copyright (C) Institute of Research Engineers and Doctors, USA .All rights reserved.

ISBN: 978-1-63248-080-4 doi: 10.15224/ 978-1-63248-080-4-73

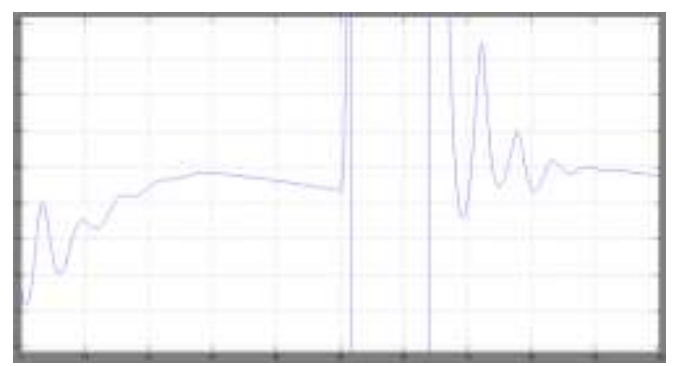

Figure 3. Dynamic traction factor without control

It may occur a sharp fluctuations in the value of $E^{\mu \alpha}$ during the change of belt speed. And its increase indicates an increase in tension of the cargo branch, and reducing tension empty branch. Therefore, it may occur sagging of the conveyor belt and slippage. The essence of the developed system of stabilization and would be to the transition speed of the belt from one level to another to change the weight of the tension device, thus to maintain traction factor at a given level. $E^{\mu \alpha}\left(G_{r y}\right)$ and $G_{r y}\left(E^{\mu \alpha}\right)$ were also found by least squares method using Matlab Control System Toolbox. Using an inverse relationship:

$$
G_{k y}\left(E^{\mu \alpha}\right)=250 \cdot\left(E^{\mu \alpha}\right)^{2}-9980 \cdot\left(E^{\mu \alpha}\right)+113380
$$

We can calculate the difference between the desired and actual traction factor ,i.e. error. The received error value of the tensioning device weight may be associated with errors of the carriage movement of tension device $s_{7}$. These data are shown in Table 2.

TABLE 2.

\begin{tabular}{|l|l|l|l|l|l|}
\hline $\begin{array}{l}G_{H y} \\
, \mathrm{~N}\end{array}$ & 30000 & 35000 & 40000 & 45000 & 50000 \\
\hline$S_{7}$ & $-1,04$ & $-1,146$ & $-1,244$ & $-1,347$ & $-1,452$ \\
& & & & & \\
\hline
\end{tabular}

\begin{tabular}{|l|l|l|l|l|l|}
\hline 52000 & 60000 & 70000 & 80000 & 90000 & 100000 \\
\hline$-1,493$ & $-1,626$ & $-1,793$ & $-1,945$ & $-2,112$ & $-2,271$ \\
& & & & & \\
\hline
\end{tabular}

Finally, there was found a relationship between the weight of the tensioning device and its movement. This relationship is linear:

$$
s_{7}\left(G_{H y}\right)=-0,0000161 \cdot\left(G_{H y}\right)-0,663
$$

Thus can vary the value of traction factor by changing the position of the carriage of tensioning device. We selected the model of the tension device movement from a general model of the belt conveyor to a separate system. The output value (tension device movement $s_{7}$ ) will be one of the external control actions for the model of the conveyor belt. The scheme for obtaining errors and implementation of traction factor control by changing the carriage position of the tensioning device is shown in Figure 4.

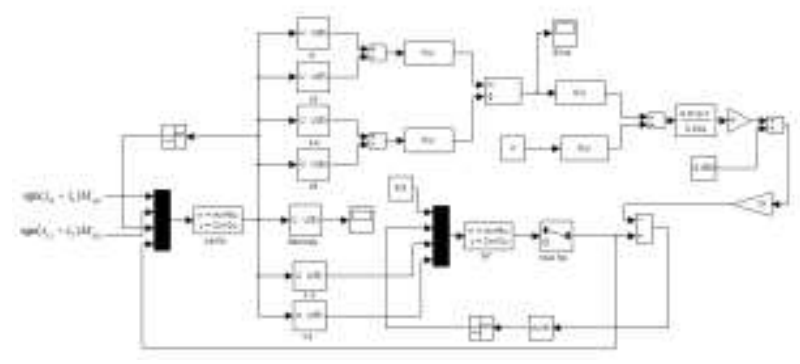

Figure 4. Scheme for obtaining errors and implementation of traction factor control by changing the carriage position of the tensioning device

If we have already mentioned the situation when the tension on the trailing branches S1 drops sharply after the increase of the rotational speed of the drive drum. While the tension on the incoming branch S4 increases, and the value of traction factors go beyond the desired level, the carriage of the tensioning device changes its position, thus avoiding a sagging tape and slippage. The results of the stabilization system shown in Figure. 5, Figure 6 and Figure 7. Changing the carriage position of the 
tensioning device occurs during the transition of the conveyor from one speed to another and due to this change is selected tape slack.

\section{$E^{\mu \alpha}$}

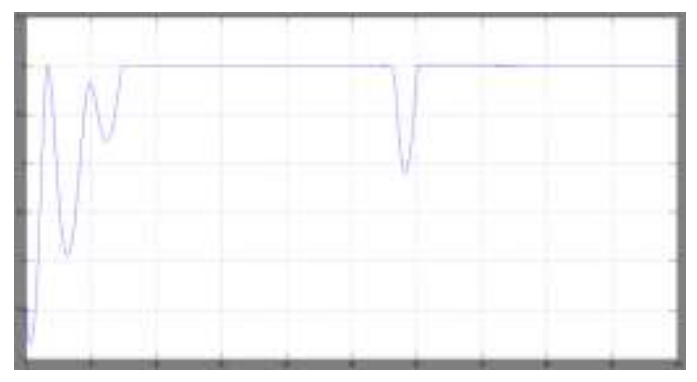

$\mathrm{t}, \mathrm{s}$

Fig.5. Dynamic traction factor control in the implementation of the carriage position of the tensioning device

$V, \mathrm{~m} / \mathrm{s}$

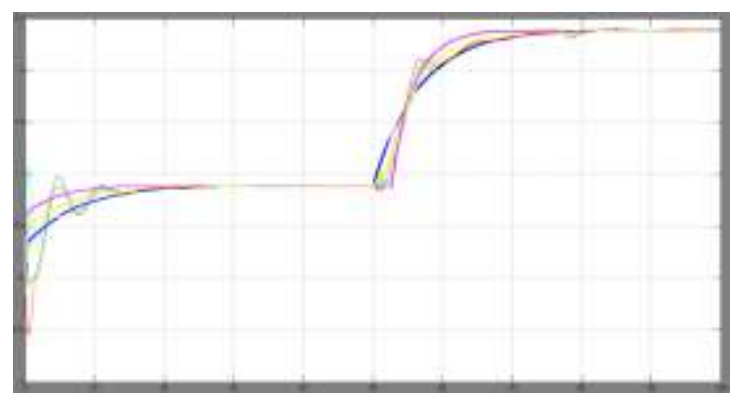

$\mathrm{t}, \mathrm{s}$

Fig.6. Transients velocity of concentrated masses of the conveyor belt in the implementation of control

$$
\delta_{7}, \mathrm{M}
$$

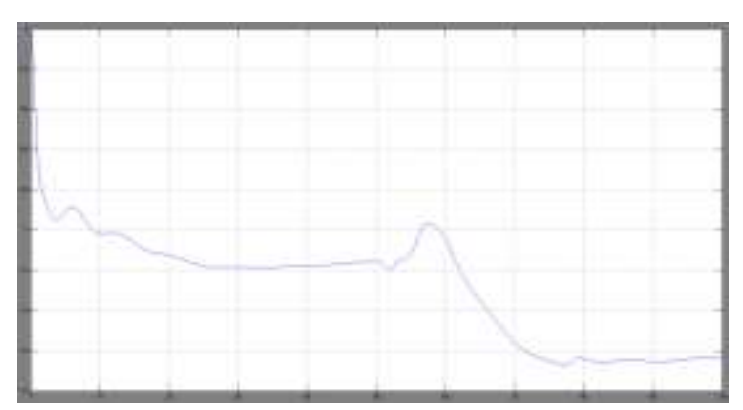

t,s
Fig.7. Movement of the tensioning device in the implementation of control

\section{Conclusion}

As can be seen from the graph in Figure 5 the value of the traction factor stabilized $E^{\mu \alpha} \leq 4$ that satisfies the requirements without slippage belt movement. Figure 6 shows a smooth joint motion of concentrated mass of the conveyor belt when moving at a constant speed and during the transition to a different speed of the conveyor. It can be concluded about the satisfactory operation of the system of stabilizing of traction factor of belt conveyor with two-engine drive.

\section{References:}

[1] Dmitrieva V.V, Development and research of system of automatic stabilization of linear load of the main conveyor. The thesis for the degree of candidate of technical sciences. M., 2005.

[2] Dmitrieva V.V, S.V Gershun "Development of a mathematical model of the conveyor belt with twoengine drive". - M .: Publishing MSMU, GIABA №8, 2008.

[3] Dmitrieva V.V, "Modern automation tasks belt", GIABA, 2014, №3, p. 65-72.

[4] Dmitrieva V.V "A mathematical model of the object as the main conveyor control and automation " «Mining machines and equipment» №7, 2001.

[5] Dmitrieva V.V, Pevzner L.D., Babitchev Y. «Automatic control of conveyor belt movement under the function of stochastic load». APCOM, 2008.

[6] Journal "Automation and operation of conveyor transport" March 2011.

[7] Journal "Mining" №4 2008. 